\title{
MANAGEMENT STRATEGIES IN A CASE OF POTT'S SPINE WITH DOWN SYNDROME- A RARE ENTITY
}

\author{
Jujhar Singh', Sarvdeep Singh Dhatt², Vishal Kumar ${ }^{3}$
}

1Orthopaedic Surgeon and Medical Officer, Central Institute of Orthopaedics (CIO), VMMC and Safdarjung Hospital, New Delhi, India. ${ }^{2}$ Additional Professor, Department of Orthopaedics, PGIMER, Chandigarh, Punjab, India.

${ }^{3}$ Assistant Professor, Department of Orthopaedics, PGIMER, Chandigarh, Punjab, India.

HOW TO CITE THIS ARTICLE: Singh J, Dhatt SS, Kumar V. Management strategies in a case of Pott's spine with down syndrome- a rare entity. J. Evolution Med. Dent. Sci. 2019;8(08):540-543, DOI: 10.14260/jemds/2019/119

\section{PRESENTATION OF CASE}

A 14 yrs. old male child, a known case of Down syndrome was referred to our center with chief complaints of gradual onset pain in mid-back region and inability to walk for the past 5 months. He also had impaired bladder and bowel control, evening rise of temperature and significant weight loss during this period. Patient was already on anti-tubercular chemotherapy for the past 2 months and was advised complete bed rest however; there was no improvement in general and clinical status as per history. There was also past history of microbiologically proven pulmonary tuberculosis 2 years back for which he took ATT for 6 months. On clinical examination, stigmata of down syndrome were noted (Moderate intellectual disability, umbilical hernia, stunted growth, short neck, anorectal malformation etc.). On local examination, angular type of kyphotic deformity was noted over T9-T10 region with para-spinal muscle spasm as well as direct and rotatory tenderness. On neurological examination, due to intellectual disability, accurate interpretation was not possible, however, there was decreased muscle bulk, positive Babinski sign, clasp knife spasticity in both the lower limbs along with exaggerated DTRs. Paraparesis was noted in both the lower limbs (Frankel Grade C) along with loss of bowel and bladder control without any involuntary movements. Sensory examination and spinal level could not be assessed accurately due to lack of patient co-operation. Nevertheless, loss of sensations below the level of umbilicus was apparent clinically.

\section{CLINICAL DIAGNOSIS}

Pott's Spine with Paraparesis And Bowel \& Bladder Involvement In 14 Yrs. Old Male Child with Associated Down Syndrome.

\section{DISCUSSION OF MANAGEMENT}

Pre-operative work-up with plain X-rays of Dorsolumbar spine revealed anterior wedge collapse and destruction at T9-T10 vertebra with kyphotic deformity of 43 degrees. There was loss of disc spaces with erosion and fuzziness of pre and para-vertebral margins (Figure 1A). Plain X-rays of chest revealed hilar nodules with fibrotic changes. On MRI, para-vertebral abscess was seen around T8-T11 region along with destruction of vertebral bodies (Figure 1B). Cord

'Financial or Other Competing Interest': None.

Submission 01-01-2019, Peer Review 09-02-2019,

Acceptance 15-02-2019, Published 25-02-2019.

Corresponding Author:

Dr. Sarvdeep Singh Dhatt,

Department of Orthopaedics,

PGIMER, Sector-12,

Chandigarh-160012, Punjab, India

E-mail:sdhatt@yahoo.com

DOI: $10.14260 /$ jemds $/ 2019 / 119$ kinking and compromise were noted around T9-T10 along with epidural abscess. On laboratory analysis, hypoproteinemia $(4.3 \mathrm{gm} / \mathrm{dl})$ was present with elevated ESR (55 $\mathrm{mm} / \mathrm{hr}$ ) and CRP (87 mg/L). On performing CT guided aspiration of para-vertebral abscess, material aspirated was found to be +ve for Acid fast bacilli (AFB). Pre-operative echocardiogram revealed valvular abnormalities with compromised ejection fraction (43\%). Pulmonary function test revealed decreased vital capacity (2.4L) and compromised pulmonary reserve. Patients relatives were explained in detail regarding the nature of disease need for surgical intervention and post-operative complications. After ensuring ventilatory back-up and thorough pre-anaesthetic check-up, plan of surgical debridement and decompression along with posterior instrumentation and fusion via posterior approach was made to minimize post-operative cardiorespiratory compromise.

Patient was placed in prone position under general anaesthesia. A posterior midline incision (Figure 2A) was used. Posterior elements including lamina, facet joint and transverse process were exposed sub-periosteally at fusion level and extra-periosteally at rest of the levels. Posterolateral extrapleural approach was used for cord decompression. Necrotic debris around the body and disc were curetted out and paraspinal abscess was drained after costo-transversectomy. Long segment pedicle screw fixation was done using 8 trans-pedicular screws in T8, 9, 11, 12. Pedicle subtraction osteotomy was done by removing wedge from T10 vertebra. Partial laminectomy was performed for circumferential decompression (Figure 2B) of spinal cord. Walls of abscess were meticulously removed, and pus was drained. Spine was shortened posteriorly under direct visualization of the cord. Deformity was further corrected with contoured rods (Figure 2C) using compression manoeuvres taking utmost care to prevent excessive stretching or distraction. Autogenous bone graft for posterior fusion was used after thorough focal debridement without compromising spinal stability. 1 gm Streptomycin was locally administered after thorough lavage. Wound was closed in layers and debrided material was sent for culture and histopathological examination.

No cardio-respiratory complication was encountered in immediate post-operative period. Drain was removed after 48 hrs. during initial wound inspection. Anti-tubercular chemotherapy was continued along with IV $3^{\text {rd }}$ generation Cephalosporin for 1 week post-operatively. Routine AP and lateral X-rays along with CT scan (Figure 3) were done postoperatively to assess graft placement and instrumentation. 12 degrees of kyphotic correction was achieved. Spine was immobilized in rigid external orthosis and patient was encouraged to sit propped-up along with wheel-chair mobilization. Sutures were removed at 3 weeks. Patient was followed every 2 weekly for the first 2 months and thereafter, 
monthly till 6 months. Plain radiographs were obtained at each follow-up visit to determine fusion status, development or progression of deformity and status of instrumentation. ESR and CRP were also assessed at each visit. Follow-up was done using Frankel grading[1] for neurological status and Modified Prolo scale[2] for functional assessment.

Tuberculosis was confirmed by bacterial culture and histopathology of intra-op specimens. Patient was continued post-operatively on anti-tubercular chemotherapy along with 6 cycles of immunomodulation therapy with levamisole 75 mg OD for 3 days followed by an interval of 7 days. Wound completely healed in 4 weeks and a decreasing trend was noted in ESR and CRP. During follow-up, gradual improvement was noted in functional, neurological and nutritional parameters. There was good functional outcome with no residual pain (P5), occasional use of NSAIDs (M5) and patient was able to work at moderate capacity (E4) with limitation of strenuous activities (F4). Bowel and bladder control as well as power in both the lower limbs were regained (Frankel Grade E) and patient was allowed ambulation with rigid spinal brace. He could walk without support and climb stairs. There was a sense of wellbeing along with gradual regaining of weight and normalization of total protein level. On follow-up X-rays, there was no loss of correction or progression of kyphosis and graft incorporated successfully without spinal instability.

Spinal tuberculosis is the most common as well as the most dangerous form of skeletal tuberculosis, comprising almost $50 \%$ of all cases[3] with associated neurological compromise in $10-30 \%$ cases. ${ }^{[4]}$ More than $90 \%$ cases of Pott's spine without neurological complications can achieve clinical healing by following "Middle Path Regime" without the need for surgical intervention, however, only 38\% cases with neurological involvement recover with ATT alone.[5] Indications for operative decompression are neurological deficit not responding to anti-tubercular chemotherapy for 34 weeks, worsening of neurological status while on ATT, advanced cases with bladder bowel involvement, pre or para vertebral abscess and significant kyphotic deformity $\left(>40^{\circ}\right)$ or instability.[6] Childhood tuberculosis is characterized by involvement of multiple vertebrae, spinal cord compression and severe kyphotic deformity. Hence, if surgical intervention is planned, prevention of post-operative deformity aggravation and long segment stabilization are the major factors determining good outcome.[7]

In children with associated down syndrome, preoperative cardio-respiratory compromise, intellectual disability and compromised cellular immunity further complicate the scenario. Immunomodulation must be considered in these patients to augment the response as well as to shorten the duration of anti-tubercular chemotherapy. ${ }^{[8]}$

Anterior approach is considered to be the operative gold standard for debridement and decompression in Pott's spine, due to advantages of less muscle dissection, direct access to pathology, protection of healthy posterior elements and the provision to place large amount of graft for fusion. $[9,10]$ However, complications include post-operative cardiorespiratory compromise, chances of increase in kyphotic deformity and hence instability. Other issues include inadequacy of anterior instrumentation to provide stability due to concomitant inflammation and infection.[11]

Posterior approach along with long segment posterior instrumentation on the other hand, is quite effective in preventing graft related complications and progression of kyphosis with added advantage of less chances of postoperative cardio-pulmonary compromise, better fixation as well as less surgical time, less bleeding, and shorter hospital stay.[12-16] Gokce et al [17] reported good outcomes in 12 patients with angular kyphotic deformity who underwent posterior decompression and corrective osteotomy with instrumentation. According to Lee et al, [18] trans-pedicular instrumentation provides rapid relief of instability and excellent prevention of long-term deformity without removal of much bone from posterior elements. In our case too, with posterior extra-pleural approach, there was no loss of correction or progression of deformity during follow up and patient regained his complete neurological status without serious complications due to pre-operatively compromised cardio-respiratory status. Staged procedures as well as combined anterior decompression and posterior instrumentation [19-21] techniques have also been described in literature, but their efficacy still remains questionable.

The complicating factors which must be kept in mind while planning the management of Pott's spine in a child with down syndrome are-

1. Complete pre-operative cardio-respiratory evaluation to assess the risk of surgery.

2. Dangers related to prolonged disease course due to compromised cellular immunity, intellectual disability precluding strict bed rest and GI related issues leading to poor nutrition and lack of compliance.

3. More chances of severe kyphotic deformity as well as multiple level vertebral involvement in children as compared to adult spinal tuberculosis.

4. Associated spinal cord abnormalities [22, 23] like ischemic cord infarction due to hypo-perfusion of anterior spinal artery along with arachnoiditis that can lead to rapid neurological de-compensation due to even minor cord compression.

Following guidelines must be adhered to while tackling these complicating factors for the most effective management and achieving good outcome in these patients-

1. Early operative management under ATT cover as opposed to conservative management.

2. Single stage posterior extra-pleural approach along with circumferential decompression without excessive removal of posterior healthy bone stock along with posterior instrumentation, deformity correction and fusion to minimize post-operative cardio-respiratory compromise.

3. Need for immunomodulation along with anti-tubercular chemotherapy due to compromised cellular immunity.

4. An experienced surgeon in a tertiary care set-up with ICU back-up. 


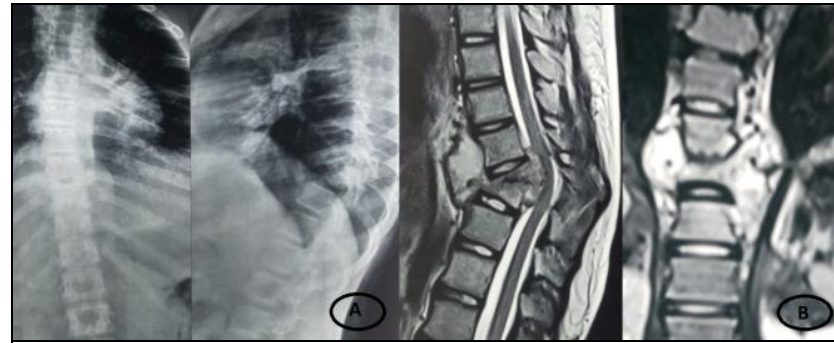

Figure 1-A. Pre-Operative Plain X-Rays Showing Destruction at T9-T10 Level Along With 43 Degrees Kyphotic Deformity B) Pre-Operative MRI Showing Cord Kinking and Compromise Along with Pre and Para Vertebral Abscess

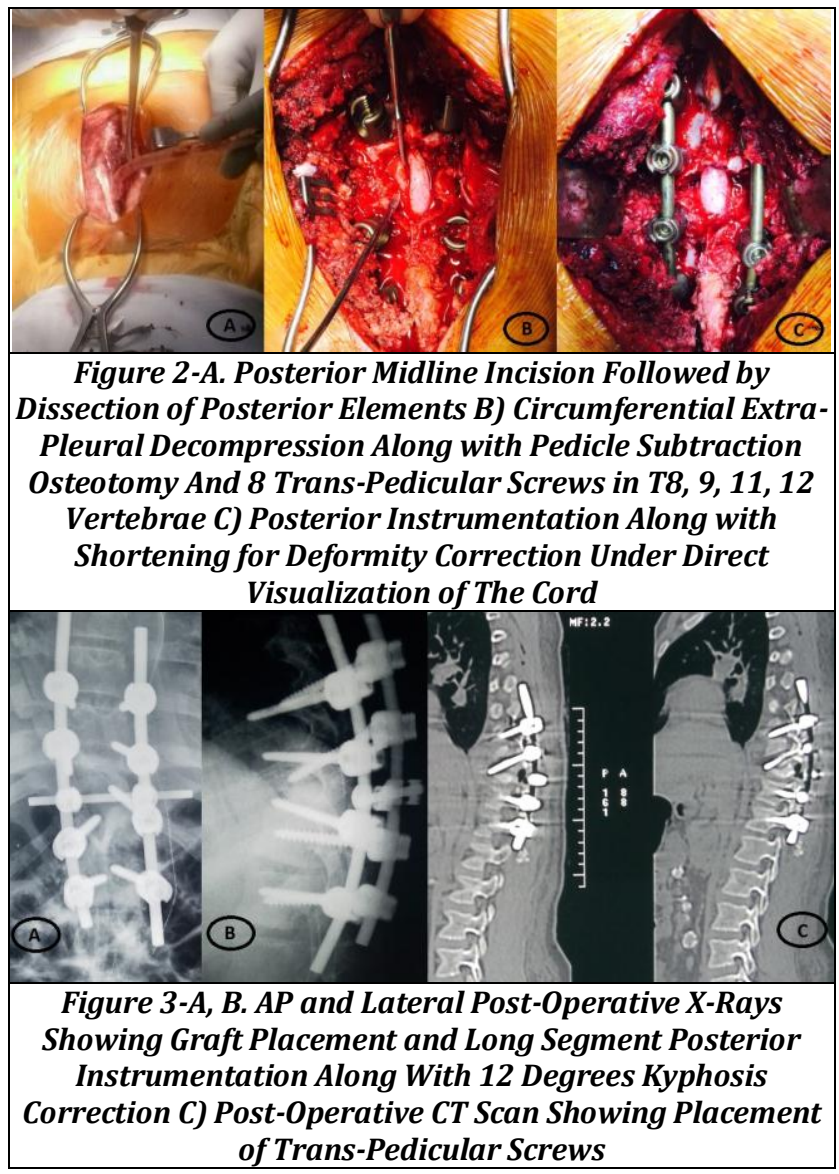

\section{FINAL DIAGNOSIS}

Pott's Spine with Neurological Involvement in A 14 Yrs. Old Child with Down Syndrome.

\section{ACKNOWLEDGEMENTS}

All authors have contributed equally to this work with no conflict of interests.

\section{REFERENCES}

[1] Frankel HL, Hancock DO, Hyslop G, et al. The value of postural reduction in the initial management of closed injuries of the spine with paraplegia and tetraplegia. I. Paraplegia 1969;7(3):179-92.

[2] Brantigan JW, Steffee AD. A carbon fiber implant to aid interbody lumbar fusion. Two-year clinical results in the first 26 patients. Spine 1993;18(14):2106-7.

[3] Rasouli MR, Mirkoohi M, Vaccaro AR, et al. Spinal tuberculosis: diagnosis and management. Asian Spine J 2012;6(4):294-308.
[4] Tuli SM. Results of treatment of spinal tuberculosis by "middle-path: regime. J Bone \& Joint Surg 1975;57(1):13-23.

[5] Tuli SM. Tuberculosis of the spine: a historical review. Clin Orthop Relat Res 2007;460:29-38.

[6] Sai Kiran NA, Vaishya S, Kale SS, et al. Surgical results in patients with tuberculosis of the spine and severe lower-extremity motor deficits: a retrospective study of 48 patients. J Neurosurg Spine 2007;6(4):320-6.

[7] Zhang HQ, Wang YX, Guo CF, et al. One-stage posterior focus debridement, fusion and instrumentation in the surgical treatment of cervicothoracic spinal tuberculosis with kyphosis in children: a preliminary report. Childs Nerv Syst 2011;27(5):735-42.

[8] Arora A, Nadkarni B, Dev G, et al. The use of immunomodulators as an adjunct to anti tuberculous chemotherapy in non-responsive patients with osteoarticular tuberculosis. J Bone Joint Surg $\mathrm{Br}$ 2006;88(2):264-9.

[9] Benli IT, Kaya A, Acaroglu E. Anterior instrumentation in tuberculous spondylitis: Is it effective and safe? Clin Orthop Relat Res 2007;460:108-16.

[10] Jain AK, Dhammi IK, Prashad B, et al. Simultaneous anterior decompression and posterior instrumentation of the tuberculous spine using an anterolateral extrapleural approach. J Bone Joint Surg Br 2008;90(11):1477-81.

[11] Krodel A, Kruger A, Lohscheidt K, et al. Anterior debridement, fusion and extrafocal stabilization in the treatment of osteomyelitis of the spine. J Spinal Disord 1999;12(1):17-26.

[12] Moon MS. Combined posterior instrumentation and anterior interbody fusion for active tuberculous kyphosis of the thoraco-lumbar spine. Curr Orthopaedics 1991;5(3):177-9.

[13] Moon MS, Woo YK, Lee KS, et al. Posterior instrumentation and anterior interbody fusion for tuberculous kyphosis of dorsal and lumbar spines. Spine (Phila Pa 1976) 1995;20(7):1910-6.

[14] Jain AK, Dhammi IK. Tuberculosis of the spine: a review. Clin Orthop Relat Res 2007;460:39-49.

[15] Güven 0, Kumano K, Yalçin $S$, et al. A single stage posterior approach and rigid fixation for preventing kyphosis in the treatment of spinal tuberculosis. Spine (Phila Pa 1976) 1994;19(9):1039-43.

[16] Sundararaj GD, Behera S, Ravi V, et al. Role of posterior stabilisation in the management of tuberculosis of the dorsal and lumbar spine. J Bone Joint Surg $\mathrm{Br}$ 2003;85(1):100-6.

[17] Gokce A, Ozturkmen Y, Mutlu S, et al. Spinal osteotomy: correcting sagittal balance in tuberculous spondylitis. J Spinal Disord Tech 2008;21(7):484-8.

[18] Lee TC, Lu K, Yang LC, et al. Transpedicular instrumentation as an adjunct in the treatment of thoracolumbar and lumbar spine tuberculosis with early stage bone destruction. J Neurosurg 1999;91(Suppl 2):163-9.

[19] Zhang HQ, Guo CF, Xiao XG, et al. One-stage surgical management for multilevel tuberculous spondylitis of the upper thoracic region by anterior decompression, 
strut autografting, posterior instrumentation and fusion. J Spinal Disord Tech 2007;20(4):263-7.

[20] Huang QS, Zheng C, Hu Y, et al. One-stage surgical management for children with spinal tuberculosis by anterior decompression and posterior instrumentation. Int Orthop 2009;33(5):1385-90.

[21] Fukuta S, Miyamoto K, Masuda T, et al. Two stage (Posterior and anterior) surgical treatment using posterior spinal instrumentation for pyogenic and tuberculotic spondylitis. Spine (Phila $\mathrm{Pa}$ 1976) 2003;28(15):E302-E8.
[22] Sohal AS, Sundaram M, Mallewa M, et al. Anterior spinal artery syndrome in a girl with Down syndrome: case report and literature review. The Journal of Spinal Cord Medicine 2009;32(3):349-54.

[23] Nance JR, Golomb MR. Ischemic spinal cord infarction in children without vertebral fracture. Pediatr Neurol 2007;36(4):209-16. 